\title{
Bacillus cereus y su implicación en la inocuidad de los alimentos. Parte I I
}

\author{
Bacillus cereus and its involvement in food safety: Part I I
}

\author{
Tamara K Martino'; Virginia Leyva'"; Yamila Puig'"'; I dalmis Hernández'v; \\ Tamara Díaz"; Maritza de los Reyes ${ }^{\mathrm{vI}}$; Ailen Camejo ${ }^{\mathrm{vI}}$
}

'Máster en Microbiología. Investigador Agregado. Instituto de Nutrición e Higiene de los Alimentos. La Habana, Cuba.

"Licenciada en Bioquímica. Investigador Auxiliar. Instituto de Nutrición e Higiene de los Alimentos. La Habana, Cuba.

IIIEspecialista de I Grado en Microbiología. Investigador Agregado. Instituto de Nutrición e Higiene de los Alimentos. La Habana, Cuba.

IV Máster en Microbiología. Instructor. Instituto de Nutrición e Higiene de los Alimentos. La Habana, Cuba.

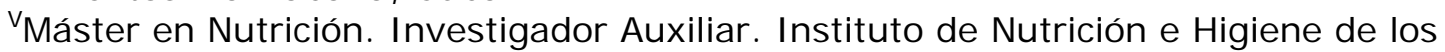
Alimentos. La Habana, Cuba.

V'Técnica A en Procesos Biológicos. Instituto de Nutrición e Higiene de los Alimentos. La Habana, Cuba.

\section{RESUMEN}

Introducción Bacillus cereus da lugar a un síndrome diarreico y a uno emético, similares a los producidos por otros microbios transmitidos por alimentos.

Objetivos Evaluar la presencia de B. cereus en alimentos asociados a brotes de origen alimentario y precisar su implicación como agente causal.

Métodos Se estudiaron 108 muestras relacionadas con 57 brotes producidos en Ciudad de La Habana desde el 2004 al 2007. La enumeración de B. cereus se realizó según la ISO 7932:2004 y la NC 38-02-12: 1988. Además se investigó: Staphylococcus coagulasa positiva (NC ISO 6888-1:2003), Clostridium perfringens (NC 38-02-11: 1988 e ISO 7937:2004) y Salmonella (ISO 6579:2002); así como, coliformes totales (NC ISO 4832:2002), coliformes fecales y Escherichia coli (NC 38-02-14: 1989).

Resultados B. cereus se aisló en $22(20,4 \%)$ muestras de alimentos de 17 brotes; en los brotes 1, 7 y 10 en más de una muestra; en los brotes 4, 6 y 17 en alimentos recién elaborados y en el 10 se confirmó en los alimentos del centro de consumo y no en los del centro de elaboración. En el brote 1 se definió como 
agente causal $\mathrm{Cl}$. perfringens y en los brotes 10 y 13 Staphylococcus coagulasa positiva, dados los niveles hallados de los microbios y la epidemiología. B. cereus se puntualizó como responsable de la enfermedad transmitida por los alimentos en 14 brotes $(24,6 \%)$. B. cereus se confirmó en platos de arroz y natilla relacionados con los brotes 10 y 4 , respectivamente.

Conclusiones B. cereus se aísla en el $20,4 \%$ de las muestras analizadas, con mayor incidencia en platos de arroz y natilla y se considera el agente responsable del $24,6 \%$ de los brotes alimentarios analizados para este microbio.

Palabras clave: Bacillus cereus, brotes de origen alimentario.

\section{ABSTRACT}

I ntroduction Bacillus cereus causes diarrheal and emetic syndromes similar to those provoked by other foodborne pathogens.

Objetives: To evaluate the presence of $B$. cereus in foodstuffs associated to outbreaks and to find out if it is the causative agent.

Methods One hundred eight suspected food samples associated to 57 outbreaks were analyzed using ISO 7932: 2004 and Cuban standard no. 38-02-12:1988. Also other microorganisms such as positive Staphylococci coagulase(Cuban standard ISO 6888-1:2003), Clostridium perfringens (Cuban standard 38-02-11: 1988 and ISO 7937:2004) and Salmonella (ISO 6579:2002) as well as total coliforms (ISO 4832: 2002), fecal coliforms and Escherichia coli (Cuban standard 38-02-14:1989).

Results B. cereus was isolated in 22 food samples from 17 outbreaks. It was confirmed in more than one sample from the outbreaks no. 1, 7 and 10; it was isolated in recently prepared foods from the outbreaks number 4, 6 and 17; however in the outbreak number 10, it was only identified in the consumption place but not in the cooking place. $\mathrm{Cl}$. perfringens was considered causative agent in outbreak number 1 and positive Staphylococci coagulase in the outbreaks no. 10 and 13, because of the levels of pathogens found and the epidemiology. B. cereus was defined as the responsible for 14 food-poisoning outbreaks; it was confirmed as causative agent in 10 and 4 outbreaks associated with the consumption of cooked rice in different ways and custard, respectively.

Conclusions B. cereus was isolated in $20.4 \%$ of the analyzed samples, being more incidental in cooked rice courses and in custards. B. cereus was responsible for $24.6 \%$ of the analyzed food-poisoning outbreaks.

Key words: Bacillus cereus, food-poisoning outbreaks.

\section{NTRODUCCI ÓN}

El papel de Bacillus cereus como causante de gastroenteritis se demostró por Hauge en Noruega en la década del ' 50 del siglo XX. ${ }^{1} \mathrm{~B}$. cereus elabora varios metabolitos extracelulares, que incluyen una enterotoxina diarreica y una emética, que se asocian a las dos formas de enfermedad transmitida por los alimentos (ETAs) que produce. ${ }^{2,3}$ 
En general se plantea que la confirmación de brotes y casos esporádicos por B. cereus, asociados al consumo de alimentos, se dificulta en ocasiones por la amplia distribución de la bacteria en el ambiente que facilita su acceso a los alimentos en bajas concentraciones, por no realizarse estudios para la búsqueda intencional de este microorganismo en el alimento y dada su similitud con los síndromes que producen Staphylococcus aureus y Clostridium perfringens. ${ }^{4,5}$

El siguiente trabajo tiene como objetivo evaluar la presencia de $\mathrm{B}$. cereus en alimentos asociados a brotes de origen alimentario y precisar la implicación del microbio como su agente causal.

\section{MÉTODOS}

En el periodo comprendido desde 2004 al 2007, se estudiaron 108 muestras asociadas a 57 brotes de origen alimentario acontecidos en centros de alimentación social de Ciudad de La Habana.

La determinación cuantitativa de B. cereus se realizó según lo recomendado por la ISO 7932 de $2004 .{ }^{6}$ Se incluyeron, además de la producción de beta hemólisis, otras pruebas fisiológicas y bioquímicas: tinción de Gram, reacción de Voges Proskauer, reducción de nitratos, fermentación y producción de gas a partir de la glucosa, licuefacción de la gelatina e hidrólisis del almidón atendiendo a lo estipulado en la norma cubana vigente NC 38-02-12 de1988. ${ }^{7}$

Paralelamente se realizó el estudio de otros microbios responsables de ETAs de acuerdo con lo establecido en las normas vigentes que se relacionan a continuación: NC ISO 6888 -1de $2003^{8}$ para determinación cuantitativa de Staphylococcus coagulasa positiva, NC 38-02-11 de $1988^{9}$ e ISO 7937 de $2004^{10}$ para determinación cuantitativa de Cl. perfringens e ISO 6579 de $2002^{11}$ para detección de Salmonella en $25 \mathrm{~g}$. A todas las muestras se les determinó, además, microorganismos coliformes totales y fecales, así como presencia de Escherichia coli, según la NC ISO 4832 de $2002^{12}$ y la NC 38-02-14 de 1989. ${ }^{13}$

\section{RESULTADOS Y DISCUSIÓN}

Se aislaron en total 22 cepas de $B$. cereus correspondientes a igual número de muestras de alimentos $(20,4 \%)$, asociadas a 17 brotes del total estudiado. Todas las cepas confirmadas como B. cereus fueron bacilos Gram positivos formadores de espora, sin esporangio distendido, que fermentaron la glucosa sin producción de gas, redujeron los nitratos a nitritos, fueron positivas en la reacción de Voges Proskauer, licuaron la gelatina, hidrolizaron el almidón ${ }^{7}$ y produjeron beta hemólisis. ${ }^{6}$

Como se observa en la tabla, en los brotes 1,7 y 10, la bacteria se confirmó en 2 de las muestras analizadas. En los brotes 4, 6, 10 y 17 donde se estudiaron las muestras testigos procedentes de los centros de elaboración o cocinas centrales y de los centros de consumo, se constató en 3 de ellos (brotes 4, 6 y 17) contaminación con el microbio en el alimento recién elaborado. 
Tabla. Resultados obtenidos en alimentos implicados en brotes de ETA con muestras positivas para B. cereus

\begin{tabular}{|c|c|c|c|c|c|}
\hline Brote & Alimento & $\begin{array}{l}\text { B. } \\
\text { cereus/g }\end{array}$ & Brote & Alimento & $\begin{array}{l}\text { B. } \\
\text { cereus/g }\end{array}$ \\
\hline \multicolumn{6}{|c|}{ Año 2004} \\
\hline \multirow[t]{3}{*}{$1 *$} & $\begin{array}{l}\text { Coditos con } \\
\text { mortadella }^{c}\end{array}$ & $\begin{array}{c}4,2 \times \\
10^{3}\end{array}$ & 3 & $\begin{array}{l}\text { Arroz con } \\
\text { leche }^{c}\end{array}$ & $\begin{array}{c}2,2 \times \\
10^{5}\end{array}$ \\
\hline & J udías ${ }^{c}$ & $<10^{2}$ & \multirow{2}{*}{4} & $\begin{array}{l}\text { Natilla } \\
\text { chocolate }^{\mathrm{e}}\end{array}$ & $3,0 \times 10^{3}$ \\
\hline & Natilla $^{c}$ & $\begin{array}{c}2,5 \times \\
10^{4}\end{array}$ & & $\begin{array}{l}\text { Natilla } \\
\text { chocolate }^{c}\end{array}$ & $3,0 \times 103$ \\
\hline \multirow[t]{3}{*}{2} & Espaguetis $^{c}$ & $<10^{2}$ & 5 & Chícharos $^{\mathrm{C}}$ & $2,0 \times 10^{3}$ \\
\hline & $\begin{array}{l}\text { Salsa de } \\
\text { tomate }^{c}\end{array}$ & $\begin{array}{c}6,3 \times \\
10^{2}\end{array}$ & \multirow{2}{*}{6} & Natilla ${ }^{e}$ & $2,0 \times 10^{3}$ \\
\hline & $\begin{array}{l}\text { Steak de } \\
\text { jamónc }\end{array}$ & $<10^{2}$ & & Natilla $^{c}$ & $<10^{2}$ \\
\hline \multicolumn{6}{|c|}{ Año 2005} \\
\hline \multirow[t]{2}{*}{7} & $\begin{array}{l}\text { Masas de } \\
\text { cerdo asado }\end{array}$ & $2,5 \times 10^{2}$ & 9 & $\begin{array}{l}\text { Arroz con } \\
\text { leche }^{c}\end{array}$ & $4,6 \times 10^{4}$ \\
\hline & Arroz congríc $^{c}$ & $2,0 \times 10^{2}$ & \multirow{4}{*}{$10 *$} & $\begin{array}{l}\text { Arroz c/ } \\
\text { embutido }\end{array}$ & $<10^{2}$ \\
\hline \multirow[t]{3}{*}{8} & $\begin{array}{l}\text { Picadillo } \\
\text { condimentado }\end{array}$ & $<10^{2}$ & & $\begin{array}{l}\text { Steak de } \\
\text { jamón }\end{array}$ & $<10^{2}$ \\
\hline & $\begin{array}{l}\text { Arroz con } \\
\text { leche }^{c}\end{array}$ & $\begin{array}{r}3,2 \\
\times 10^{4}\end{array}$ & & $\begin{array}{l}\text { Arroz c/ } \\
\text { embutido }\end{array}$ & $\begin{array}{c}4,8 \times \\
10^{4}\end{array}$ \\
\hline & & & & $\begin{array}{l}\text { Steak de } \\
\text { jamón }{ }^{c}\end{array}$ & $3,3 \times 10^{4}$ \\
\hline \multicolumn{6}{|c|}{2006} \\
\hline 11 & Natilla $^{c}$ & $7,0 \times 10^{5}$ & & & \\
\hline \multicolumn{6}{|c|}{ Año 2007} \\
\hline \multirow[t]{2}{*}{12} & Arroz con leche & $\begin{array}{c}1,5 \times \\
10^{6}\end{array}$ & 15 & $\begin{array}{l}\text { Arroz } \\
\text { amarilloc }\end{array}$ & $1,5 \times 10^{6}$ \\
\hline & & & & $\begin{array}{l}\text { Pollo } \\
\text { marinado }\end{array}$ & $<10^{2}$ \\
\hline \multirow[t]{3}{*}{$13^{*}$} & Sopa de pollo ${ }^{c}$ & $<10^{2}$ & \multirow{3}{*}{16} & $\begin{array}{l}\text { Sopa de } \\
\text { vegetales }\end{array}$ & $<10^{2}$ \\
\hline & Arroz amarillo ${ }^{c}$ & $<10^{2}$ & & $\begin{array}{l}\text { Arroz con } \\
\text { mariscos }\end{array}$ & $4,8 \times 10^{4}$ \\
\hline & $\begin{array}{l}\text { Pollo en } \\
\text { fricaséc }^{c}\end{array}$ & $3,2 \times 10^{4}$ & & & \\
\hline \multirow[t]{3}{*}{14} & Sopa de pollo ${ }^{c}$ & $<10^{2}$ & \multirow{3}{*}{17} & $\begin{array}{l}\text { Arroz } \\
\text { blancoe }\end{array}$ & $1,5 \times 10^{6}$ \\
\hline & Arroz blanco $^{c}$ & $1,5 \times 10^{6}$ & & $\begin{array}{l}\text { Potaje de } \\
\text { frijoles }^{\mathrm{e}}\end{array}$ & $<10^{2}$ \\
\hline & Pollo en salsac & $<10^{2}$ & & Arroz & $1,5 \times 10^{6}$ \\
\hline
\end{tabular}




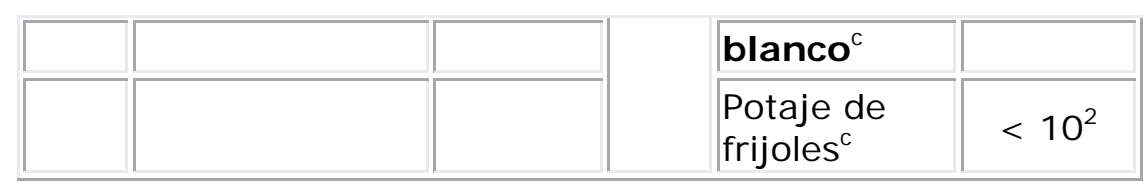

c alimento procedente del centro de consumo, ealimento procedente del centro de elaboración,

* aislamiento de otro microbio, Pres: presencia

En el brote 6 solo se aisló la bacteria en la muestra tomada del centro de elaboración. En el brote 10 a diferencia, se aisló en los 2 alimentos del centro de consumo y no en los del centro de elaboración. En las restantes 86 muestras, la concentración fue $<10^{2} \mathrm{~B}$. cereus/g y no se aisló ningún otro microbio estudiado.

En 96 de las muestras analizadas, incluyendo todas en las que se aisló algún microorganismo, las concentraciones de coliformes totales y fecales fueron $>1,5 \times 10^{5}$ microorganismos/g. E. coli se aisló en los coditos con mortadella del brote 1 , en el steak de jamón del brote 2 , en la natilla del centro de consumo asociada al brote 4 y en el arroz con leche del brote 9 .

El estudio de otros microbios develó en el brote 1, contaminación de los coditos con mortadella con $\mathrm{Cl}$. perfringens por encima de $1,5 \times 10^{5} / \mathrm{g}$. En las muestras del brote 10 se aisló Staphylococcus coagulasa positiva en concentraciones de $4,3 \times 10^{3} / \mathrm{g}$ en el arroz con embutido y de $1,8 \times 10^{3} / \mathrm{g}$ en el steak de jamón procedentes del centro de elaboración y de más de $1,5 \times 10^{6} / \mathrm{g}$ en ambos alimentos procedentes del centro de consumo. Este mismo microbio se aisló en concentraciones superiores a $1,5 \times 10^{6} / \mathrm{g}$ en el pollo relacionado con el brote 13 .

Como refleja la figura 1 , en el $20,4 \%$ de las muestras se aisló B. cereus. El hecho de hallar en los brotes 1, 7 y 10 la bacteria en más de una muestra analizada, hace sospechar que se pudo haber incurrido en una contaminación cruzada lo cual unido a las concentraciones de coliformes totales y fecales encontrados, así como al aislamiento de $\mathrm{E}$. coli en varias muestras, reveló una mala calidad sanitaria en la mayoría de los alimentos estudiados, indudablemente debido a incumplimiento de las Buenas Prácticas de Elaboración y Manipulación de los alimentos.

En el brote 6, en particular, a partir de la encuesta epidemiológica, se encontró a la natilla como alimento sospechoso de ocasionar los síntomas. Sin embargo, en la muestra tomada del centro donde enfermaron los comensales no se aisló B. cereus; aunque sí en la tomada del centro donde se elaboraron los alimentos. Los microorganismos no se distribuyen de manera homogénea en los alimentos sólidos o semisólidos, por lo que pueden contaminar y colonizar zonas específicas y alcanzar altas concentraciones, precisamente esta es una de las causas por las que no siempre todos los individuos que consumen un alimento dado enferman, también podría explicar por qué en alimentos estudiados de otros brotes no se aisló este u otro microbio en cuestión, si se asume que las muestras testigos conservadas en las entidades, son representativas de cada lote de alimento elaborado y ofertado.

En los brotes 4 y 17 se aisló B. cereus, ya desde las muestras testigo procedentes del centro de elaboración. En el brote 10, a diferencia, solo se aisló la bacteria en los alimentos del centro de consumo. La causa de la presencia de B. cereus en alimentos asociados a enfermedades de origen alimentario, usualmente se debe a 
manejos inadecuados después de cocinarlos. En estos episodios las esporas logran sobrevivir al proceso de cocción, lo cual ocurre sobre todo cuando se emplean grandes recipientes, y después de la germinación las células vegetativas resultantes se multiplican durante la conservación inadecuada del alimento. ${ }^{1}$ La mayoría de las descripciones de los brotes de ETAs señalan como causas los efectos o prácticas no correctas en los establecimientos donde se sirven o preparan alimentos y en los propios hogares. ${ }^{14}$

Estas malas prácticas se asocian, usualmente, con la aplicación de inadecuada temperatura de conservación de los alimentos elaborados hasta su consumo (por debajo de $70 \stackrel{\circ}{\circ}$ para alimentos que se sirven calientes o temperaturas superiores a los $5{ }^{\circ} \mathrm{C}$ para los refrigerados), por un tiempo prolongado (más de $2 \mathrm{~h}$ ), así como a la ocurrencia de contaminación cruzada por el empleo de utensilios sin la higiene adecuada para su manipulación, lo cual demuestra la violación de las Buenas Prácticas de Higiene y permite el crecimiento del microbio sin que se altere el alimento en cuestión.

Los platos a base de arroz y la natilla, fueron los alimentos con mayor incidencia del microbio, con aislamientos en muestras de los brotes de 10 y 4, respectivamente (tabla). Resultados similares se obtuvieron en la primera parte de este trabajo al analizar este tipo de alimentos en platos terminados no asociados a brotes de origen alimentario; por lo que se ratifica lo necesaria que es la educación y entrenamiento de los manipuladores de alimentos como forma más adecuada para reducir este problema sanitario. ${ }^{15}$

En el brote 1, se aisló más de un microbio en los alimentos analizados. El síndrome diarreico causado por $\mathrm{B}$. cereus y la ETAs de $\mathrm{Cl}$. perfringens son difíciles de diferenciar, dadas sus manifestaciones clínicas y período de incubación; en la mayoría de los casos se tiende a pensar en uno u otro agente como causante del brote por el tipo de alimento implicado, su forma de preparación y condiciones de conservación.

Se considera que para que aparezca la enfermedad clínica por $\mathrm{Cl}$. perfringens, al igual que para $B$. cereus, se requiere que el alimento consumido contenga $10^{5} \mathrm{o}$ más microorganismos/g. ${ }^{3}$ La mayor implicación de esta bacteria anaerobia en brotes de origen alimentario viene dado también porque las esporas sobreviven a las temperaturas normales de cocción, germinan y se multiplican durante el enfriamiento lento, el almacenamiento a temperatura ambiente o el recalentamiento inadecuado. ${ }^{2}$

En el brote 1, el hecho de haber aislado $\mathrm{B}$. cereus y $\mathrm{Cl}$. perfringens en 2 y 1 muestra respectivamente, de los 3 alimentos implicados, hace más firme el criterio de que no se cumplieron las medidas higiénico-sanitarias adecuadas en el proceso de elaboración, conservación y manipulación de los alimentos.

No fue posible realizar estudios que permitieran determinar la enterotoxina presente en los alimentos, pero a partir de las concentraciones de los microbios que se obtuvieron y la epidemiología del brote, se definió a $\mathrm{Cl}$. perfringens como su agente causal.

En los brotes 10 y 13, igualmente se aislaron 2 microbios en los alimentos implicados. En el primer brote los aislamientos se obtuvieron en los 2 alimentos provenientes tanto del centro de elaboración como del de consumo, con concentraciones más elevadas en este último, lo que indica posibles incumplimientos en la adecuada cocción y en la relación tiempo/temperatura 
durante la transportación y conservación de los alimentos antes de ser servidos; así como en su manipulación, por la ocurrencia de contaminación cruzada incluso en los alimentos recién elaborados. En el brote 13 los microbios se hallaron en el pollo en fricasé y el alimento se consumió en el mismo lugar donde se elaboró, por lo que el análisis que se deriva es muy similar.

En estos 2 brotes primó entre los síntomas el vómito con un período de incubación de alrededor de $6 \mathrm{~h}$, por lo que se asoció al síndrome emético causado por B. cereus, sin descartar la posibilidad de una intoxicación estafilocóccica. En ambos casos el microorganismo responsable de las ETAs tiene que producir una enterotoxina que es termoestable; en la intoxicación estafilocóccica, en particular, el período de incubación suele ser más corto, en ocasiones de 30 min a 4 h después de ingerido el alimento conteniendo la exotoxina. ${ }^{3}$ Las elevadas concentraciones de Staphylococcus coagulasa positiva en el arroz con embutido y en el steak de jamón involucrados en el brote 10 y del pollo asociado al brote 13, superiores a las de B. cereus, llevaron a considerarlo como agente responsable de los síntomas en ambos brotes. Los estafilococos coagulasa positiva incluyen a Staphylococcus aureus y otras especies de este género que coagulan el plasma, que también son capaces de producir enterotoxinas y por tanto pueden dar origen a ETAs. ${ }^{3,8}$

Staphylococcus coagulasa positiva, es el segundo agente causal de brotes en Cuba. Muchos alimentos pueden albergar esta bacteria, especialmente aquellos proteicos, como los productos cárnicos, los pescados, la leche, las cremas y las ensaladas; ${ }^{3}$ se encuentra generalmente en nariz y en garganta, así como en las manos y en las uñas y se puede localizar en el $50,0 \%$ de las muestras clínicas aisladas de personas no enfermas, de ahí su fácil acceso a cualquier alimento que requiera de manipulación. La tos, los estornudos y las heridas infectadas de los manipuladores, pueden ser un mecanismo de contaminación. ${ }^{2}$

En estos tres brotes $(1,10$ y 13$)$ la epidemiología, apoyada por los resultados obtenidos en el laboratorio, permitió cerrar los brotes. La determinación de la enterotoxina responsable de los síntomas, a partir del alimento o de la cepa del microorganismo ofrecería un resultado más fidedigno en estos estudios.

En los otros 14 brotes $(24,6 \%)$ donde se aisló $B$. cereus en uno o varios de los alimentos implicados (tabla) se definió a este microbio como responsable de las ETAs. En estos brotes la enfermedad se caracterizó por diarreas, ocasionalmente se informó algún caso de fiebre o vómito con períodos de incubación entre 9 y 12 h; en estos estudios se investigó también $\mathrm{Cl}$. perfringens y Salmonella, pero no se obtuvo ningún hallazgo. A pesar de que en algunas muestras la concentración de $B$. cereus encontrada no superó o alcanzó la concentración $10 \%$ g considerada necesaria para que ocurran ETAs ${ }^{3}$ (fig. 1) , existen estudios publicados que confirman que este microbio es responsable de brotes en concentraciones similares a las halladas en esta investigación. ${ }^{2,5,15}$ Además, debe tenerse en cuenta que en las instalaciones donde se elaboran los alimentos, en ocasiones, la muestra testigo se colecta incorrectamente, lo que implica que se refrigeren estos alimentos prácticamente una vez concluido el proceso de cocción o al inicio de comenzar el servicio, factor que conlleva a la detención del crecimiento microbiano, y entonces las concentraciones del microorganismo aislado no reflejan el grado de contaminación real que llega al consumidor.

La capacitación a los manipuladores de alimentos y a las administraciones de las instalaciones de alimentación social es vital para evitar incumplimientos en las prácticas de higiene que puedan propiciar la ocurrencia de brotes de ETAs por B. cereus. 
La figura 2 muestra el número total de brotes estudiados en Ciudad de La Habana por año con el número de brotes en los que se sospechó B. cereus como agente causal. Se tomaron en cuenta los datos aportados por el Departamento de Microbiología de Alimentos del Instituto de Nutrición e Higiene de los Alimentos. De estos, se confirmaron en el 2004 , el $26,4 \%$; en el 2005 , el $17,6 \%$; en el 2006 , el $16,7 \%$ y en el 2007 , el $33,3 \%$. En general, se consideró que este microbio era responsable de las ETAs, al menos, en el 6,9 \% de los brotes analizados en Ciudad de La Habana del 2004 al 2007.

En conclusión, B. cereus se aisló en el 20,4 \% de las muestras de alimentos analizadas, con mayor incidencia en platos de arroz y en natilla. B. cereus se consideró el agente responsable del $24,6 \%$ de los brotes de origen alimentario analizados para este microbio.

\section{REFERENCI AS BI BLI OGRÁFICAS}

1. ICMSF. Bacterias productoras de enfermedades transmitidas por los alimentos. En: Microorganismos de los Alimentos I. Técnicas de análisis microbiológico. 2 da ed. Zaragoza (España): Acribia S.A.; 2000.p.36-7.

2. Fernández Escartín E. Inocuidad de los alimentos. México: Universidad Autónoma de Querétaro,2000: 143-51.

3. Benenson AS, editor. Control Communicable Diseases Manual. 16th ed. APHA; 1995.

4. Jonson EA. Bacillus cereus foods poisoning. En: Food borne diseases. San Diego California: Academia Press, Inc.; 1990. p. 127-35.

5. Martínez NE, Pérez J, Rodríguez M, Peregrina R. Bacillus cereus. En: Torres Vitela, Refugio M, editores. Agentes patógenos. Guadalajara, Jalisco: Universidad de Guadalajara;2002.p.69-86.

6. ISO 7932:2004. Microbiology of food and animal feeding stuffs - Horizontal method for the enumeration of presumptive Bacillus cereus - Colony-count technique at $30^{\circ} \mathrm{C}$.

7. NC 38-02-12. Sistema de normas sanitarias de alimentos. Determinación cuantitativa de Bacillus cereus. Método de análisis microbiológico; 1988.

8. NC ISO 6888-1. Microbiología de alimentos de consumo humano y animal. Método horizontal para la enumeración de Staphylococcus coagulasa positiva (Staphylococcus aureus y otras especies). Parte 1: técnica utilizando el medio Agar Baird Parker. (ISO 6888-1: 1999, IDT); 2003.

9. NC 38-02-11. Sistema de normas sanitarias de alimentos. Determinación cuantitativa de Clostridium perfringens. Método de análisis microbiológico; 1988.

10. ISO 7937. Microbiology of food and animal feeding stuffs. Horizontal method for the enumeration of Clostridium perfringens. Colony-count technique; 2004. 
11. ISO 6579. Microbiology-General guidance on methods for the detection of Salmonella;2002.

12. NC ISO 4832. Microbiología de alimentos de consumo humano y animal. Guía general para la enumeración de coliformes. Técnica de placa vertida. (ISO 4832: 1991, IDT); 2002.

13. NC 38-02-14. Sistema de normas sanitarias de alimentos. Determinación cuantitativa de coliformes fecales. Métodos de ensayos microbiológicos; 1989.

14. ICMSF. Ecología microbiana de los alimentos 2. Productos alimenticios. Vol II. Zaragoza (España): Acribia S.A.; 1985.p.739-58.

15. ICMSF. Microorganismos de los Alimentos 2. Métodos de muestreo para análisis 2da ed. Zaragoza (España): Acribia S.A.; 1999.

Recibido: 5 de enero de 2009.

Aprobado: 20 de marzo de 2009.

Tamara K Martino. Instituto de Nutrición e Higiene de los Alimentos. Dpto.

Microbiología de los Alimentos. Infanta 1158 e/ Llinás y Clavel. Centro Habana. La Habana, Cuba.

E-mail: tamara.martino@infomed.sld.cu 


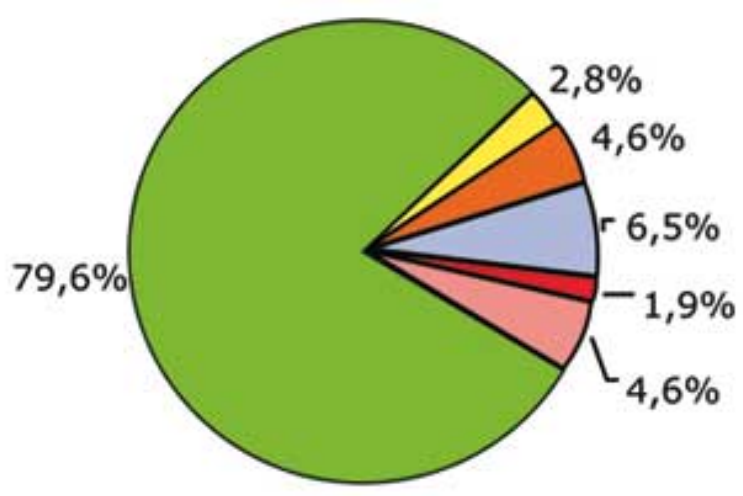

Microorganismo/g de alimento
$\square 32000-48000$
$\square<100$
$\square 220000-700000$
$\square 200-630$
1500000 y más
$\square 2000-3000$

Fig.1. Porcentajes de aislamiento de $B$. cereus según los recuentos obtenidos en muestras de brotes de origen alimentario.

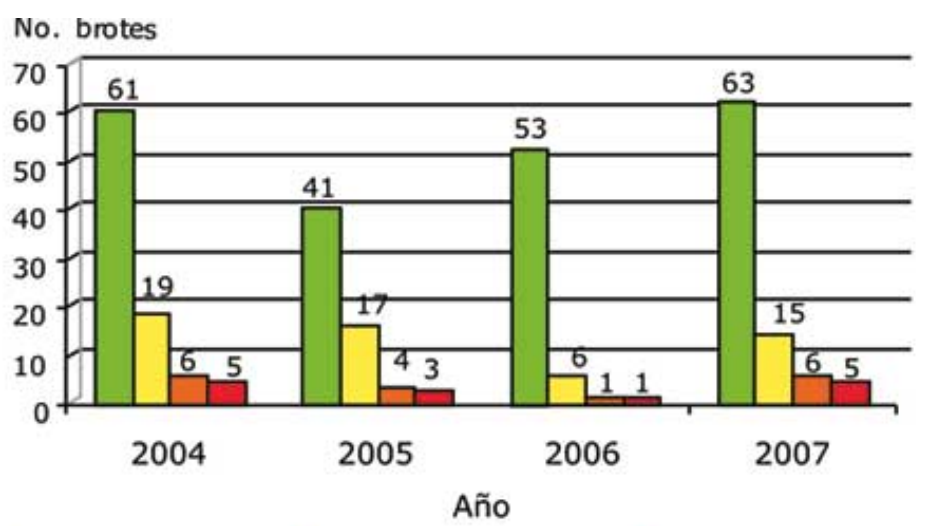

$\square$ Total $\square$ Analizados para Bc $\square$ Aislamiento de Bc $\square$ Confirmados para Bc

Fig. 2. Número de brotes de origen alimentario en Ciudad Habana confirmados para B. cereus (Bc). 